\title{
ARTÍCULOS
}

Sometido 26.10.2015. Aprobado 18.12.2015

Evaluado por el sistema double blind review. Editor Científico: Eric Cohen

DOI: http://dx.doi.org/10.1590/So034-759020160303

\section{REANALIZANDO LA COMPETITIVIDAD EN LA INDUSTRIA DEL FÚTBOL: DIFERENCIA ACUMULADA DE PUNTOS}

\author{
Reanalisando a competitividade na indústria do futebol: Diferença acumulada \\ de pontos
}

\author{
Reanalyzing the competitiveness in football leagues: Accumulated points \\ difference
}

\begin{abstract}
RESUMEN
En la presente investigación se plantea un nuevo modelo de análisis del equilibrio en las ligas de fútbol a través del Accumulated Points Difference (APD). Se examinan ocho temporadas de nueve campeonatos entre 2006-2007 y 2013-2014: Bundesliga (Alemania), Campeonato Brasileiro (Brasil), Liga BBVA (España), Ligue 1 (Francia), Eredivise (Holanda), Premier League (Inglaterra), Serie A (Italia), Primeira Liga (Portugal) y Premier League (Rusia). Posteriormente, se comparan estadísticamente los torneos a través de un análisis de varianza ANOVA one way con post hoc de Tukey. Se valida el método al confrontarlo con dos fórmulas tradicionales: Herfindahl Index of Competitive Balance (HICB) y $\mathrm{C}_{4}$ Index of Competitive Balance ( $\left.\mathrm{C}_{\mathrm{I}} \mathrm{CB}\right)$. Como resultados, se evidencia que el Campeonato Brasileiro es el torneo más equilibrado y no se aprecian diferencias estadísticamente significativas entre las ligas europeas.
\end{abstract}

PALABRAS CLAVE | Balance competitivo, Campeonato Brasileño, diferencia acumulada de puntos, fútbol profesional, economía del deporte.

\section{RESUMO}

O presente estudo objetivou criar um novo modelo de análise do balanço competitivo em ligas de futebol através do Accumulated Points Difference (APD). A amostra foi composta por nove torneios durante oito temporadas consecutivas (2006-2007 e 2013-2014): Bundesliga, Campeonato Brasileiro, Liga BBVA, Ligue 1, Eredivise, English Premier League, Serie A, Primeira Liga e Russian Premier League. Posteriormente, compararam-se estatisticamente os resultados encontrados através de uma análise de variância ANOVA one way com post hoc de Tukey. Validou-se o método ao compará-lo com duas fórmulas tradicionais: Herfindahl Index of Competitive Balance (HICB) e $\mathrm{C}_{4}$ Index of Competitive Balance (C4ICB). Como resultado, evidenciou-se o Campeonato Brasileiro como o torneio mais equilibrado, assim como não se observou diferenças significativas entre as ligas europeias.

PALAVRAS-CHAVE I Balanço competitivo, Campeonato Brasileiro, diferença acumulada de pontos, futebol profissional, economia do esporte.

THADEU GASPARETTO

tgasparetto@uvigo.es

Estudiante de doctorado de la Universidad de Vigo, Facultad de Ciencias Empresariales y Turismo Vigo, España

\section{ANGEL BARAJAS}

abarajas@uvigo.es

Profesor de la Universidad de Vigo, Facultad de Ciencias Empresariales y Turismo - Vigo, España

\begin{abstract}
The aim of the paper is to elaborate an alternative measure to compare football leagues based on Accumulated Points Difference (APD). The sample includes eight seasons (2006-2007 to 2013-2014) from nine football leagues: German Bundesliga, Campeonato Brasileiro (Brazil), La Liga BBVA (Spain), French Ligue 1, Dutch Eredivise, English Premier League, Italian Serie A, Portuguese Primeira Liga and Russian Premier League. We have employed the ANOVA one way with Tukey post hoc to compare the results. We have confirmed the robustness of the model comparing it with two traditional measures: Herfindahl Index of Competitive Balance (HICB) and $\mathrm{C}_{4}$ Index of Competitive Balance (C4ICB). As a result, we have found that the Brazilian League was the most balanced tournament in this period and there are no statistical differences between European leagues.
\end{abstract}

KEYWORDS / Competitive balance, Brazilian League, accumulated points difference, professional football, sports economics. 


\section{INTRODUCCIÓN}

En 1956, el prestigioso estudio de Rottenberg remarcó la incertidumbre del resultado deportivo como un punto clave para mantener el interés de los aficionados. Posteriormente, Neale (1964) añadió que los ingresos de un deportista o club deportivo dependerían de la calidad del adversario. Bajo esos supuestos se ha desarrollado gran parte de la literatura de Economía del Deporte. Dentro de esta área, el análisis de la competitividad de ligas deportivas ha sido uno de los temas más estudiados.

Al analizar la Major League Baseball (MLB), Levin, Mitchell, Volcker, y Will (2000) identificaron el balance competitivo como la posibilidad de todos los equipos para clasificarse a los playoffs al comienzo de la temporada. Por su parte, Rodríguez (2012) ha adaptado esa definición a la realidad del fútbol europeo, midiendo el equilibrio competitivo mediante la posibilidad de todos los clubes para clasificarse para disputar la UEFA Champions League (UCL).

El primer modelo de análisis del balance competitivo fue desarrollado por El-Hodiri y Quirk (1971). Luego, vinieron los destacados estudios de Vrooman (1995), Hausman y Leonard (1997) y Késenne (2000), entre muchos otros. Sin embargo, dichos autores no consideraron en sus modelos la compra-venta de jugadores y algunos incluso supusieron funciones de ingresos iguales para todos los equipos. Por lo tanto, se investigó en un contexto muy distinto del fútbol.

Debido a las características anteriormente citadas, diversos autores han elaborado nuevos indicadores para el cálculo del balance competitivo en otros deportes profesionales. También se han adaptado algunos ratios de concentración al escenario deportivo. Entre los más usados, se destacan el Índice $\mathrm{C}_{5}$ de Michie y Oughton (2004); el Top 3, elaborado por Goossens (2006); el Competitive Balance Ratio (CBR), desarrollado por Humphreys (2002); el Coeficiente de Gini, tradicionalmente usado para medir las desigualdades de riqueza entre países; y el Índice de Herfindahl e Hirschman ( $\mathrm{HHI})$, que mide la concentración económica en un mercado. Sin embargo, Fort y Maxcy (2003) destacaron que no existe un método ideal de análisis de la competitividad, debido a que cada fórmula permite evaluar el equilibrio deportivo desde diferentes perspectivas.

Zimbalist (2002) afirmó que el balance competitivo podría ser medido a través de dos aspectos: el nivel de concentración y el nivel de dominio. Además, también se evalúa la competitividad de ligas deportivas en tres dimensiones temporales. Según Cairns, Jennett, y Sloane (1986), se considera el balance competitivo a corto plazo el análisis partido a partido; el medio plazo se refiere a la competitividad dentro de una determinada temporada, como por ejemplo las disputas por el título o el descenso; y a largo plazo estaría relacionado al dominio de los clubes entre temporadas. Los estudios de Booth (2004), Eckard (1998), Goossens (2006), Humphreys (2002), Michie y Oughton (2004) y Pawlowski, Breuer, y Hovemann (2010) han centrado sus análisis en el largo plazo. La fórmula UCS measure, elaborada por Janssens y Késenne (1987) y modificada por Pawlowski y Anders (2012), mide el equilibrio a medio plazo. Mientras tanto, Peel y Thomas (1988) destacaron las apuestas deportivas como medida de análisis de la incertidumbre en partidos individuales y, posteriormente, Peel y Thomas (1992) aplicaron por primera vez al fútbol el Índice de Theil (Theil, 1967), también empleado por Buraimo y Simmons (2008) y Czarnitzki y Stadtmann (2002), investigando el balance competitivo a corto plazo. Evans (2014) presenta una interesante revisión de gran parte de los modelos desarrollados para el análisis del balance competitivo hasta ahora.

Además de la diversidad de modelos, también se reflejan las distintas maneras de medir el desempeño deportivo en la literatura. Uno de los métodos más tradicionales es el porcentaje de victorias. Usado por primera vez por Noll (1988) y seguido por Scully (1989), también se observa su utilización en los artículos de Fort (2007), Quirk y Fort (1991), Trandel y Maxcy (2011) y Vrooman (1996). Sin embargo, resulta una medida más adecuada para los deportes estadounidenses donde no hay empates. El fútbol sí presenta esa posibilidad. Esto implica que el porcentaje de victorias no sea la medida más adecuada. Koning (2000) y Michie y Oughton (2004) emplean la concentración de puntos de un grupo de equipos sobre el total de puntos del torneo. Curran, Jennings, y Sedgwick (2009), Goosens (2006) y Szymanski y Kuypers (1999) analizaron el dominio de los clubes, considerando la clasificación final, a lo largo de varias temporadas. Conjuntamente, uno de los problemas encontrados en ese tipo de análisis es la diferencia en el número de participantes en cada liga. Así, algunos estudios como los de Koning (2000), Michie y Oughton (2004) y Owen, Ryan, y Weatherspoon (2007) han ajustados sus modelos en función de las diferencias de cada liga para poder compararlas.

El estudio del balance competitivo de las ligas de fútbol resulta relevante pues un mayor equilibrio deriva en mayor interés por parte de los aficionados. Esto se traduce en mejores asistencias y más audiencia televisiva (Dobson \& Goddard, 2011; Forrest \& Simmons, 2002). Además, el análisis del balance competitivo en ligas deportivas proporciona hallazgos importantes para la toma de decisiones sobre políticas deportivas como: topes salariales (Fort \& Quik, 1995; Szymanski, 2003), reparto de ingresos (Budzinski, 2012; Szymanski, 2003) y Financial Fair Play (Franck, 2014; Müller, Lammert, \& Hovemann, 2012; Peeters \& Szymanski, 2014; Preuss, Haugen, \& Schubert, 2014; Szymanski, 2014). Por lo tanto, siendo el deporte una industria en permanente 
crecimiento y la gestión deportiva uno de los destacados temas en la administración contemporánea, se aprecia indispensable investigar la competitividad dentro de una liga deportiva.

Gran parte de los estudios sobre el balance competitivo deriva de la teoría clásica propuesta por Rottenberg (1956) que puso de manifiesto que los aficionados se interesan más por los torneos equilibrados. En el presente artículo se ha buscado analizar el balance competitivo desde una perspectiva diferente. En los principales torneos de fútbol, la puntuación final de cada equipo define la tabla de clasificación. Así, el club que más puntos logra es el campeón, los mejores se clasifican a los torneos internacionales y los últimos descienden a la categoría inferior. Por lo tanto, para que se apliquen los conceptos de competitividad de Levin et al. (2000) y Rodríguez (2012) debería existir equilibrio entre todos los clubes. De esa manera, se supone que el torneo más equilibrado será aquel en el que la diferencia de puntos entre los competidores sea la menor posible, pues esto refleja mayores posibilidades de obtener el título o clasificarse para torneos continentales por parte de todos los clubes durante una temporada. Vistas estas consideraciones, el objetivo de la presente investigación ha sido crear un modelo para comparar torneos de fútbol y analizar el equilibrio existente entre las ligas usando como herramienta la diferencia acumulada de puntos entre los competidores.

\section{METODOLOGÍA}

El presente estudio ha adoptado una metodología empírica, proponiendo un modelo matemático para la comparación de ligas de fútbol con distinto número de participantes.

\section{El modelo}

El modelo ha sido desarrollado basado en la diferencia acumulada de puntos entre todos los participantes. Este indicador, propuesto por primera vez en este trabajo, calcula la suma de las diferencias de puntos entre los participantes. Estas diferencias se computan disminuyendo del total de puntos del campeón lo que ha conseguido el clasificado en segundo puesto. Se repite sucesivamente hasta la diferencia de puntos del penúltimo con respecto al último de la tabla de clasificación. Sin embargo, como se propone comparar ligas con distinto número de participantes, se ha ajustado la fórmula por el máximo desequilibrio posible, adaptando los conceptos de Goossens (2006) y Owen (2010). Se resalta que el modelo se aplica a ligas de fútbol que tengan la siguiente puntuación: 3 puntos por victoria, 1 por empate y o por derrota.
Considerando las características definidas anteriormente, se analiza el balance competitivo simulando una presunta liga en formato doble Round-Robin con dos clubes participantes: A y $B$. Se ha elegido dicho modelo pues es lo predominante en las grandes ligas europeas (Goossens \& Spieksma, 2012). El desequilibrio máximo se produce con el hecho de que uno de los clubes (A) gane los dos partidos y el otro (B) no gane ninguno. Así, el equipo A alcanzaría la puntuación máxima (seis puntos) y el B la mínima (cero).

Conjuntamente, se simula una liga con un participante más: A, B y C. El máximo desequilibrio resultaría cuando un equipo (A) venza a todos sus partidos (cuatro), sumando al final del torneo doce puntos. El segundo puesto (B) ganaría dos partidos y totalizaría seis puntos y el último (C) perdería todos sus partidos y no puntuaría. Una vez más se observa que seis puntos separarían cada puesto en la tabla de clasificación final.

En la Tabla 1 se presenta la simulación del máximo desequilibrio en un torneo con veinte clubes participantes.

\section{Tabla 1. Máximo desequilibrio en una Liga Round-Robin duplo con veinte participantes}

\begin{tabular}{|c|c|c|c|c|c|c|}
\hline Puesto & Club & V & $\mathbf{E}$ & D & Puntos & Diferencia \\
\hline 1 & A & 38 & 0 & 0 & 114 & \\
\hline 2 & $B$ & 36 & 0 & 2 & 108 & 6 \\
\hline 3 & C & 34 & 0 & 4 & 102 & 6 \\
\hline 4 & $D$ & 32 & 0 & 6 & 96 & 6 \\
\hline 5 & $E$ & 30 & 0 & 8 & 90 & 6 \\
\hline 6 & $\mathrm{~F}$ & 28 & 0 & 10 & 84 & 6 \\
\hline 7 & $\mathrm{G}$ & 26 & 0 & 12 & 78 & 6 \\
\hline 8 & $\mathrm{H}$ & 24 & 0 & 14 & 72 & 6 \\
\hline 9 & I & 22 & 0 & 16 & 66 & 6 \\
\hline 10 & $J$ & 20 & 0 & 18 & 60 & 6 \\
\hline 11 & $\mathrm{~K}$ & 18 & 0 & 20 & 54 & 6 \\
\hline 12 & $\mathrm{~L}$ & 16 & 0 & 22 & 48 & 6 \\
\hline 13 & $M$ & 14 & 0 & 24 & 42 & 6 \\
\hline 14 & $\mathrm{~N}$ & 12 & 0 & 26 & 36 & 6 \\
\hline 15 & 0 & 10 & 0 & 28 & 30 & 6 \\
\hline 16 & $P$ & 8 & 0 & 30 & 24 & 6 \\
\hline 17 & Q & 6 & 0 & 32 & 18 & 6 \\
\hline 18 & $\mathrm{R}$ & 4 & 0 & 34 & 12 & 6 \\
\hline 19 & $\mathrm{~S}$ & 2 & 0 & 36 & 6 & 6 \\
\hline \multirow[t]{2}{*}{20} & $\mathrm{~T}$ & 0 & 0 & 38 & 0 & 6 \\
\hline & & & & & & 114 \\
\hline
\end{tabular}

Note: $\mathrm{V}=$ victoria, $\mathrm{E}=$ empate, $\mathrm{D}=$ derrota 
Como se observa, el concepto de máximo desequilibrio se aplica a todos los torneos doble Round-Robin, independiente del número de participantes. Además, cualquier resultado distinto de lo presentado en la Tabla 1 cambiaría la competitividad en esta liga, suponiendo mayor equilibrio.

Así, el cálculo del desequilibrio máximo seria el siguiente:

$$
\text { Unbalance }_{\text {máx }}=6 \text { * }(N-1) \quad \text { (1) }
$$

De esa manera, se presenta a continuación la fórmula creada del Accumulated Points Difference (APD):

$$
A P D=\left(\frac{\sum_{i=1}^{N} T P_{i}-T P_{i+1}}{\text { Unbalance }_{\max }}\right){ }_{100}
$$

Dónde $N$ es el número de equipos participantes y TP el total de puntos de cada club $i$ al final del torneo.

\section{Muestra}

Se compara el balance competitivo, medido por la APD, de las nueve ligas de fútbol: la Bundesliga alemana, el Campeonato Brasileiro, la Liga BBVA en España, la Ligue 1 francesa, la Eredivise holandesa, la Premier League inglesa, la Serie A italiana, la Primeira Liga de Portugal y la Premier League rusa. Se han analizado ocho temporadas de cada torneo: de 2006-2007 hasta la temporada 2013-2014 en los torneos europeos y del año 2006 hasta 2013 en Brasil. Se ha elegido dichos campeonatos en virtud de que se rigen por un similar modelo de competición: Round-Robin duplo. Los datos han sido recogidos en la página web de la Rec.Sport. Soccer Statistics Foundation (http://www.rsssf.com/histdom.html).

\section{Análisis}

Como se ha explicado anteriormente, se calcula el ADP a través de un ratio entre el total de puntos acumulados en cada liga y su máximo desequilibrio posible. Así, se interpreta como el porcentaje de desequilibrio existente en cada uno de los torneos. Por lo tanto, los resultados encontrados demuestran las diferencias del balance competitivo en cada torneo investigado.

Haan, Koning, y Witteloostuijn (2007) destacan la posibilidad de utilizar métodos estadísticos estándar en los análisis del balance competitivo. Por esto, se ha realizado un análisis de varianza ANOVA one way con post hoc de Tukey, buscando encontrar si existen diferencias significativas entre las medias de los distintos torneos durante el período investigado. Dicha técnica estadística se ha llevado a cabo tras realizar los diagnósticos previos de normalidad de distribución (Shapiro-Wilk) y homogeneidad de varianza (Levene).

\section{Dispersión de los puestos}

Posteriormente al análisis del APD, se ha evaluado el comportamiento de los clubes en la tabla de clasificación de las ligas investigadas. Para eso, se ha generado un gráfico de la dispersión de los puestos medios de cada participante con el objetivo de observar la concentración de clubes o grupos de equipos dominantes en cada uno de los torneos. Como los campeonatos concluyen con el ascenso y descenso de equipos, aquellos que no participaron en la primera división en alguna temporada se les ha adjudicado el puesto $N+1$.

\section{Confrontación con modelos tradicionales}

Como el objetivo de la investigación ha sido crear un nuevo modelo de análisis del balance competitivo, es necesaria la comparación del Accumulated Poins Difference con otros indicadores tradicionales. Para eso, se ha considerado el Campeonato Brasileiro como escenario y se ha confrontado los siguientes modelos: Herfindahl Index of Competitive Balance ( $\mathrm{HICB})$ y $\mathrm{C}_{4}$ Index of Competitive Balance (C4ICB) - adaptación del $\mathrm{C}_{5}$ ICB de Michie y Oughton (2004) empleado por Drummond, Araújo, y Shikida (2010).

El HICB es una adaptación del Herfindahl-Hirschman Index $(\mathrm{HHI})$, usado en la administración para medir el nivel de concentración de empresas en un mercado (Barajas, 2010). El resultado se presenta entre o y 1 , dónde el valor 1 representaría el monopolio y el o significaría un número infinito de agentes. Según Michie y Oughton (2004), el HHI es sensible a las variaciones en el número de participantes en una liga deportiva, así que lo ajustaron para la comparación de distintas ligas.

El C4ICB ha sido un ajuste de dos fórmulas preliminares. Koning (2000) desarrolló en $C R_{k}$, un ratio entre el total de puntos de $k$ equipos sobre el máximo de puntos posibles por estos clubes. Posteriormente, Michie y Oughton (2004) propusieron el $C_{5}$ Ratio, en lo cual se medía la concentración de puntos de los cinco mejores clubes sobre el total de puntos de la liga. Luego, los mismos autores (Michie \& Oughton, 2004) han adaptado el modelo para ajustarlo a un número diferente de competidores, basados en el máximo equilibrio posible en cada campeonato, creando así el $C_{4}$ Index of Competitive Balance. Las fórmulas se presentan a continuación:

$$
H I C B=\left(\frac{\sum_{i=1}^{N} S_{i}^{2}}{1 / N}\right) \star 100
$$

Donde $s_{i}$ representa los puntos del equipo $i$ sobre el total de puntos de la liga y $N$ el número de clubes participantes.

$$
C_{4} I C B=\left(\frac{C_{4} \text { ratio }}{4 / N}\right) * 100
$$

(4) 
Donde $\mathrm{C}_{4}$ es el ratio entre la suma del total de puntos de los cuatro mejores clubes sobre el total de puntos de la liga y $\mathrm{N}$ representa el número de equipos participantes.

En ambos índices el equilibrio máximo está representado por el valor 100, así que cuanto mayor es el resultado - más lejos de 100 - más desequilibrada ha sido la competición.

\section{RESULTADOS Y DISCUSIÓN}

En la Tabla 2 se presentan el ADP de las ligas investigadas.

Tabla 2. Accumulated Points Difference de las ligas nacionales de Alemania, Brasil, España, Francia, Holanda, Inglaterra, Italia, Portugal y Rusia

\begin{tabular}{c|c|c|c|c|c|c|c|c|c}
\hline & \multicolumn{10}{c}{ Accumulated Points Difference (APD) } \\
\hline Temporada & Alemania & Brasil & España & Francia & Holanda & Inglaterra & Italia & Portugal & Rusia \\
\hline 2006 & 43,14 & 43,86 & 42,11 & 41,23 & 56,86 & 53,51 & 62,28 & 52,22 & 52,22 \\
\hline 2007 & 46,08 & 52,63 & 51,75 & 48,25 & 44,12 & 66,67 & 48,25 & 58,89 & 47,78 \\
\hline 2008 & 40,20 & 35,09 & 47,37 & 47,37 & 50,00 & 50,88 & 47,37 & 52,22 & 43,33 \\
\hline 2009 & 45,10 & 31,58 & 57,02 & 48,25 & 69,61 & 58,77 & 46,49 & 61,11 & 58,89 \\
\hline 2010 & 45,10 & 37,72 & 57,89 & 49,12 & 56,86 & 41,23 & 50,88 & 67,78 & 53,33 \\
\hline 2011 & 56,86 & 35,09 & 73,68 & 42,11 & 55,88 & 56,14 & 54,39 & 62,22 & 45,56 \\
\hline 2012 & 68,63 & 41,23 & 57,89 & 47,37 & 51,96 & 56,14 & 57,02 & 61,11 & 50,00 \\
\hline 2013 & 63,73 & 49,12 & 57,02 & 57,89 & 41,18 & 49,12 & 67,54 & 55,56 & 48,89 \\
\hline APD & 51,10 & 40,79 & 55,59 & 47,70 & 53,31 & 54,06 & 54,28 & 58,89 & 50,00 \\
\hline
\end{tabular}

Al observar la media del APD $\left(\mathrm{APD}_{\text {med }}\right.$ ), se percibe que el Campeonato Brasileiro ha sido, entre todos los investigados, el torneo con mejor balance competitivo en promedio durante las ocho temporadas analizadas. Una manera de comparar el balance competitivo de los torneos es a través de un análisis gráfico. Así, se presenta a continuación en el Gráfico 1 el diagrama de caja con los valores del APD de cada liga.

Gráfico 1. Diagrama de caja del Accumulated Points Difference de las ligas nacionales de Alemania, Brasil, España, Francia, Holanda, Inglaterra, Italia, Portugal y Rusia

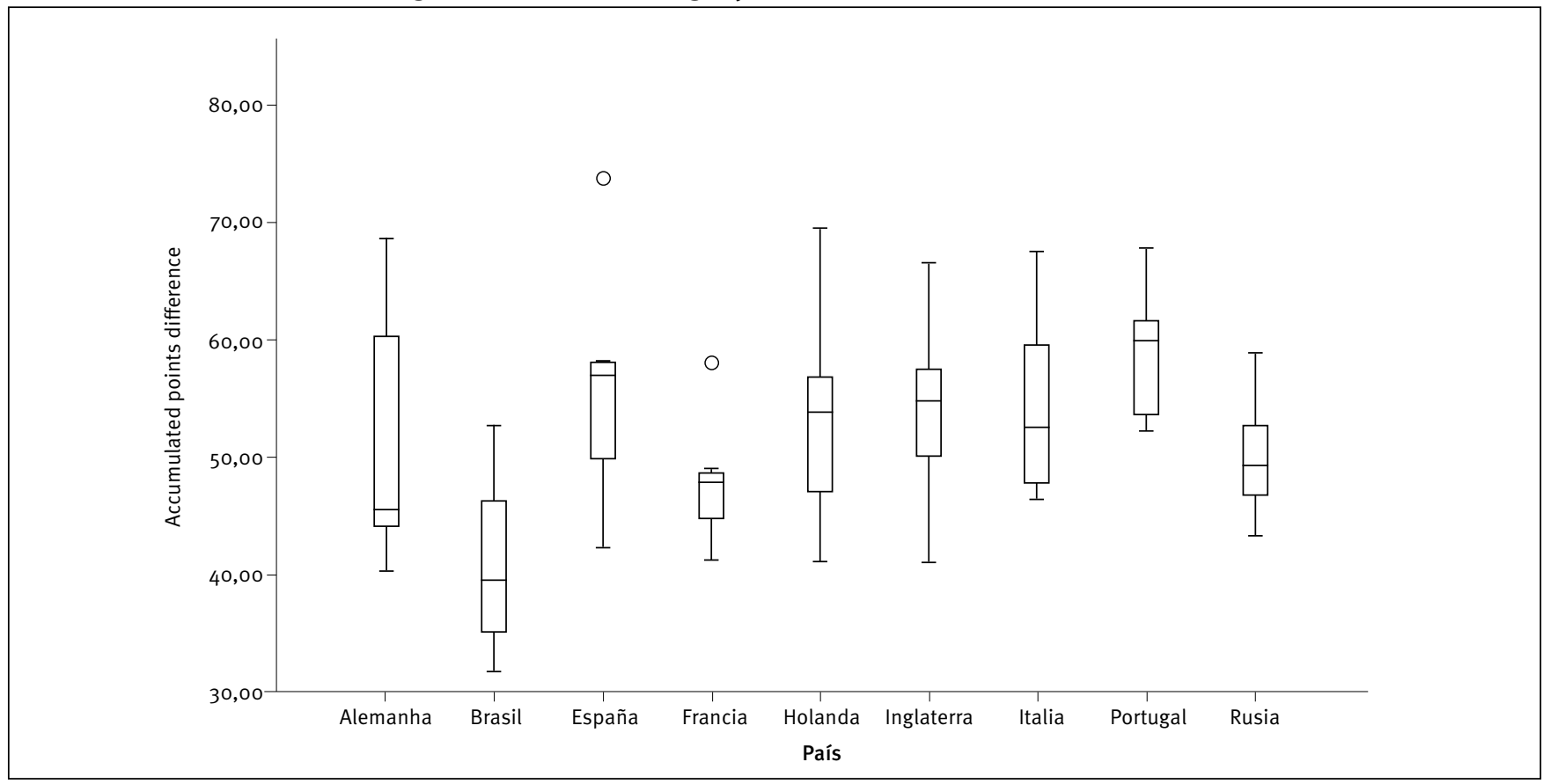


Se aprecia de forma clara que el Campeonato Brasileiro es el más equilibrado entre las ligas investigadas. Entre los torneos europeos no se observa mucha diferencia, aunque Francia y Rusia presenten mejores valores. Al mismo tiempo, es necesario resaltar la presencia de dos outliers en el Gráfico 1: uno en España y otro en Francia. Ese valor atípico en la Liga BBVA se produjo en la temporada 2011-2012, cuando el desequilibrio fue notable. En esa ocasión, el equipo campeón alcanzó los 100 puntos y la diferencia del segundo con respecto al tercero fue de treinta puntos, remarcando un gran desequilibrio. Ya en la Ligue
1 francesa, ese outlier representa la temporada 2013-2014. En ese año, el Paris Saint Germain obtuvo su segundo título consecutivo reafirmando su supremacía deportiva y financiera en el fútbol francés tras la compra del club por inversores extranjeros.

En el Gráfico 1, se observa la gran variabilidad de valores del APD dentro de cada liga. Además, analizándolo junto con la Tabla 1, se nota que el balance competitivo cambia por completo en cada temporada. Así, esa dinámica se representa a continuación en el Gráfico 2 usando como ejemplos, por motivos de claridad, solo las ligas de Alemania, Brasil y Holanda.

\section{Gráfico 2. Gráfico de líneas del Accumulated Points Difference de las ligas nacionales de Alemania, Brasil y Holanda}

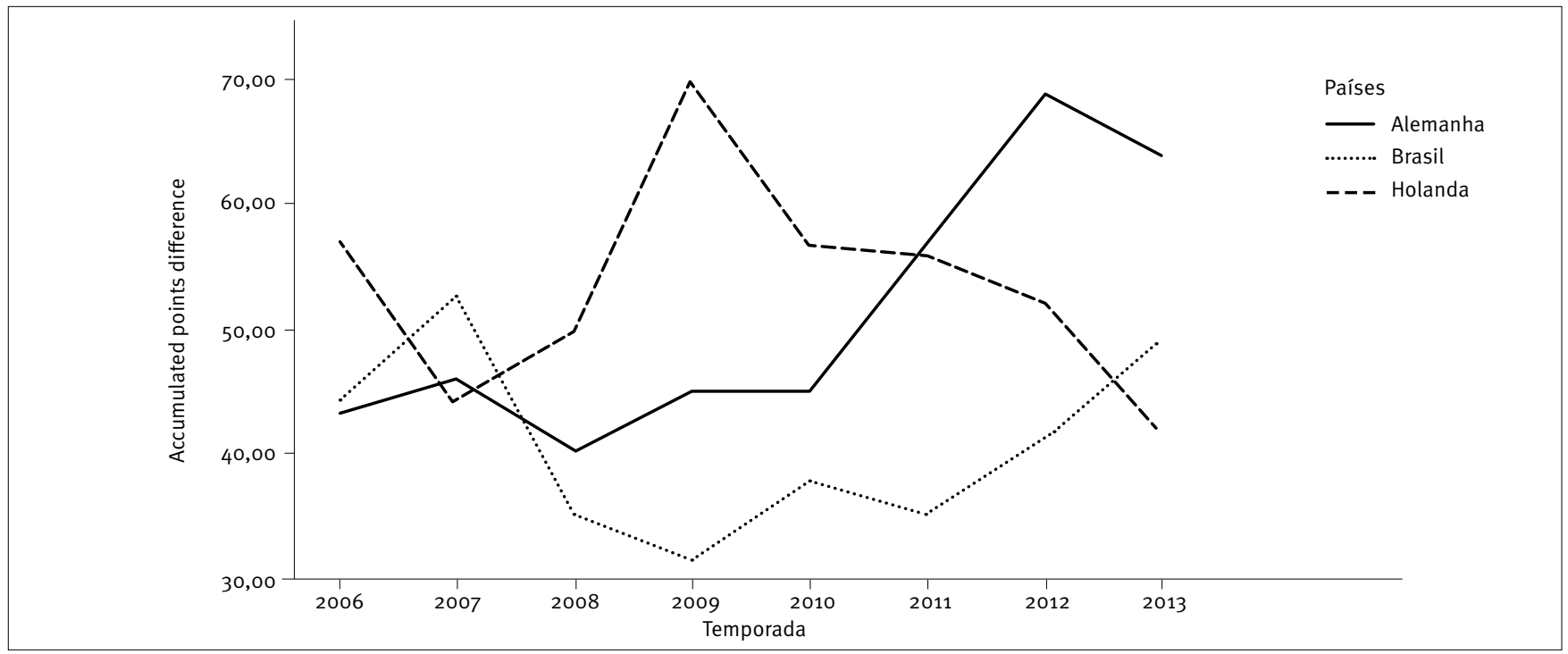

A través del gráfico de líneas se muestran los cambios ocurridos en cada temporada en el balance competitivo de los campeonatos investigados. Aunque el Campeonato Brasileiro haya sido el más equilibrado durante el período investigado, se observa que su desequilibrio se acentuó en las últimas dos temporadas. Igualmente, se observa que entre 2006 y 2010 la Bundesliga alemana ostentaba un ADP de aproximadamente 45, pero la competitividad ha disminuido notablemente en las últimas ediciones. Por otro lado, se nota que la Eredivise holandesa ha mejorado su balance competitivo en los últimos años.

Aparte de los análisis anteriormente presentados, en la presente investigación también se compararon estadísticamente los valores de APD. En primer lugar, se ha analizado la distribución de los datos y la homogeneidad de las varianzas. La prueba de Shapiro-Wilk confirma la distribución normal de los datos (ver Tabla 3). Se ha empleado dicha prueba debido al reducido número de observaciones en cada grupo. Como se observa en la Tabla 3, se ha confirmado la distribución normal de los datos en todos los torneos. La prueba de Levene confirma la homogeneidad de las varianzas (ver Tabla 4). Una vez realizadas ambas pruebas, se ha llevado a cabo el análisis de varianza ANOVA one way con Post hoc de Tukey y sus resultados se encuentran en las Tablas 5 y 6.

Tabla 3. Prueba de normalidad de Shapiro-Wilk

\begin{tabular}{c|l|c|c|c}
\hline \multirow{2}{*}{} & \multicolumn{3}{c}{ Shapiro-Wilk } \\
\cline { 2 - 5 } & Alemania & 0,856 & 8 & 0,110 \\
\hline \multirow{4}{*}{ ADP } & Brasil & 0,945 & 8 & 0,658 \\
\cline { 2 - 5 } & España & 0,912 & 8 & 0,367 \\
\cline { 2 - 5 } & Francia & 0,869 & 8 & 0,148 \\
\cline { 2 - 5 } & Holanda & 0,947 & 8 & 0,683 \\
\cline { 2 - 5 } & Inglaterra & 0,978 & 8 & 0,954 \\
\cline { 2 - 5 } & Italia & 0,912 & 8 & 0,366 \\
\cline { 2 - 5 } & Portugal & 0,935 & 8 & 0,561 \\
\cline { 2 - 5 } & Rusia & 0,977 & 8 & 0,947 \\
\hline
\end{tabular}


Tabla 4. Prueba de homogeneidad de varianzas de Levene

\begin{tabular}{c|c|c|c}
\hline Prueba de Levene & df1 & df2 & Sig. \\
\hline 1,303 & 8 & 63 & 0,258 \\
\hline
\end{tabular}

Tabla 5. Resultado del ANOVA one way

\begin{tabular}{c|c|c|c|c|c}
\hline & Suma de cuadrados & Df & Cuadrado medio & Z & Sig. \\
\hline Entre grupos & 1759,184 & 8 & 219,898 & 0,001 \\
\hline Intra grupos & 3644,709 & 63 & 57,853 & & \\
\hline Total & 5403,893 & 71 & & & \\
\hline
\end{tabular}

El nivel de significancia (o,001) pone de manifiesto que existen diferencias estadísticamente significativas entre el balance competitivo de las ligas investigadas. En la Tabla 6 se presentan los resultados del test post hoc de Tukey.

Tabla 6. Post hoc de Tukey

\begin{tabular}{|c|c|c|c|c|c|c|}
\hline (I) Liga & (J) Liga & $\begin{array}{c}\text { Diferencia media } \\
(\mathrm{I}-\mathrm{J})\end{array}$ & Error standard & Sig. & \multicolumn{2}{|c|}{ Intervalo de confianza $99 \%$} \\
\hline \multirow{5}{*}{ Brasil } & España & $-14,80263^{\star \star \star}$ & 3,80304 & 0,007 & $-29,1684$ & $-0,4368$ \\
\hline & Italia & $-13,48684^{\star \star}$ & 3,80304 & 0,020 & $-27,8526$ & 0,8789 \\
\hline & Francia & $-6,90789$ & 3,80304 & 0,671 & $-21,2737$ & 7,4579 \\
\hline & Portugal & $-18,0994^{\star \star \star}$ & 3,80304 & 0,000 & $-32,4652$ & $-3,7336$ \\
\hline & Rusia & $-9,21053$ & 3,80304 & 0,291 & $-23,5763$ & 5,1553 \\
\hline \multirow{5}{*}{ España } & Brasil & $14,80263^{* \star *}$ & 3,80304 & 0,007 & 0,4368 & 29,1684 \\
\hline & Inglaterra & 1,53509 & 3,80304 & 1,000 & $-12,8307$ & 15,9009 \\
\hline & Italia & 1,31579 & 3,80304 & 1,000 & $-13,0500$ & 15,6816 \\
\hline & Portugal & $-3,29678$ & 3,80304 & 0,994 & $-17,6626$ & 11,0690 \\
\hline & Rusia & 5,59211 & 3,80304 & 0,865 & $-8,7737$ & 19,9579 \\
\hline \multirow{8}{*}{ Inglaterra } & Brasil & $13,26754^{\star \star}$ & 3,80304 & 0,023 & $-1,0982$ & 27,6333 \\
\hline & España & $-1,53509$ & 3,80304 & 1,000 & $-15,9009$ & 12,8307 \\
\hline & Italia & $-0,21930$ & 3,80304 & 1,000 & $-14,5851$ & 14,1465 \\
\hline & Francia & 6,35965 & 3,80304 & 0,761 & $-8,0061$ & 20,7254 \\
\hline & Alemania & 2,95408 & 3,80304 & 0,997 & $-11,4117$ & 17,3199 \\
\hline & Holanda & 0,74819 & 3,80304 & 1,000 & $-13,6176$ & 15,1140 \\
\hline & Portugal & $-4,83187$ & 3,80304 & 0,936 & $-19,1977$ & 9,5339 \\
\hline & Rusia & 4,05702 & 3,80304 & 0,977 & $-10,3088$ & 18,4228 \\
\hline \multirow{2}{*}{ Italia } & Portugal & $-4,61257$ & 3,80304 & 0,951 & $-18,9784$ & 9,7532 \\
\hline & Rusia & 4,27632 & 3,80304 & 0,968 & $-10,0895$ & 18,6421 \\
\hline
\end{tabular}


Tabla 6. Post hoc de Tukey

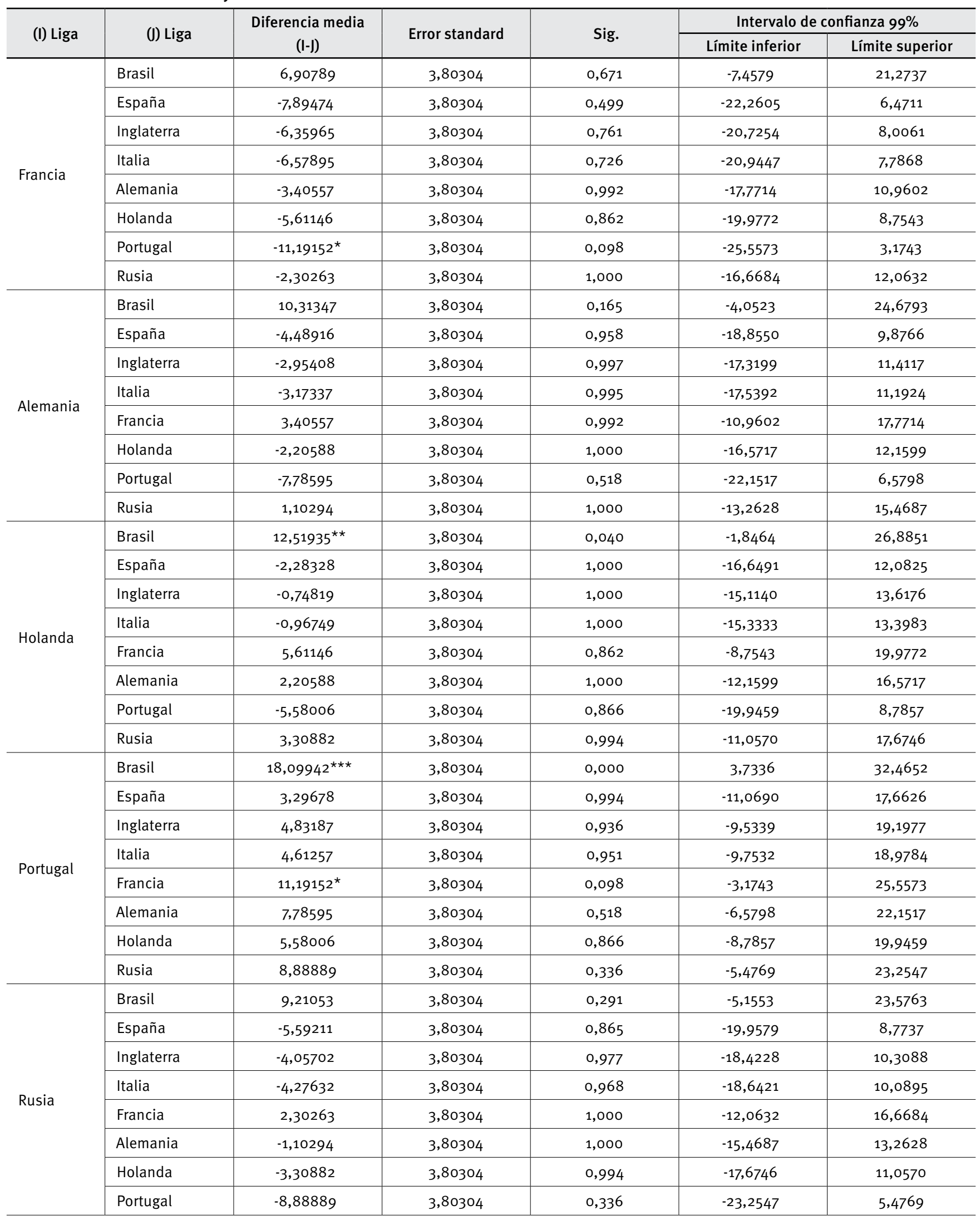

Notas: $\left(^{* * *}\right)$ La diferencia media es significativa a nivel 0,01. $\left(^{\star *}\right)$ La diferencia media es significativa a nivel 0,05. $\left(^{\star}\right)$ La diferencia media es significativa a nivel 0,10. 
La relación intra grupo, presentada en la Tabla 6, evidencia las diferencias estadísticamente significativas encontradas entre las ligas. El Campeonato Brasileiro, el torneo de mejor balance competitivo considerando el valor absoluto del ADP, se ha confirmado distinto a nivel de $1 \%$ de la Liga BBVA española y de la Primeira Liga portuguesa, como también más equilibrado que los torneos nacionales de Inglaterra, Italia y Holanda ( $p$ $0,05)$. No obstante, no se ha notado diferencia estadísticamente significativa con relación a las ligas de Alemania, Francia y Rusia. Conjuntamente, todos los torneos europeos han presentado un nivel de equilibrio similar al considerar sus varianzas, con la única excepción siendo la Ligue 1 francesa más equilibrada que la Primeira Liga portuguesa $(\mathrm{p}<0,10)$.
De manera complementaria a los hallazgos anteriores, en el Gráfico 3 se presentan los puestos medios de todos los equipos que han jugado en las ligas investigadas entre las temporadas 2006-2007 y 2013-2014. Se ha desarrollado ese gráfico con el objetivo de observar el comportamiento de los clubes durante el período investigado. Como se ha explicado en la metodología, debido al diferente número de participantes en cada liga, se ha adoptado puesto $N+1$ a los clubes que hayan disputado alguna categoría inferior a la primera división de sus países. De cualquier modo, se enfatiza la observación en los puestos más altos - más próximos al 1 - buscando encontrar clubes o grupos de equipos dominantes en cada una de las ligas investigadas.

Gráfico 3. Dispersión de los puestos medios de los equipos entre 2006-2007 y 2013-2014.

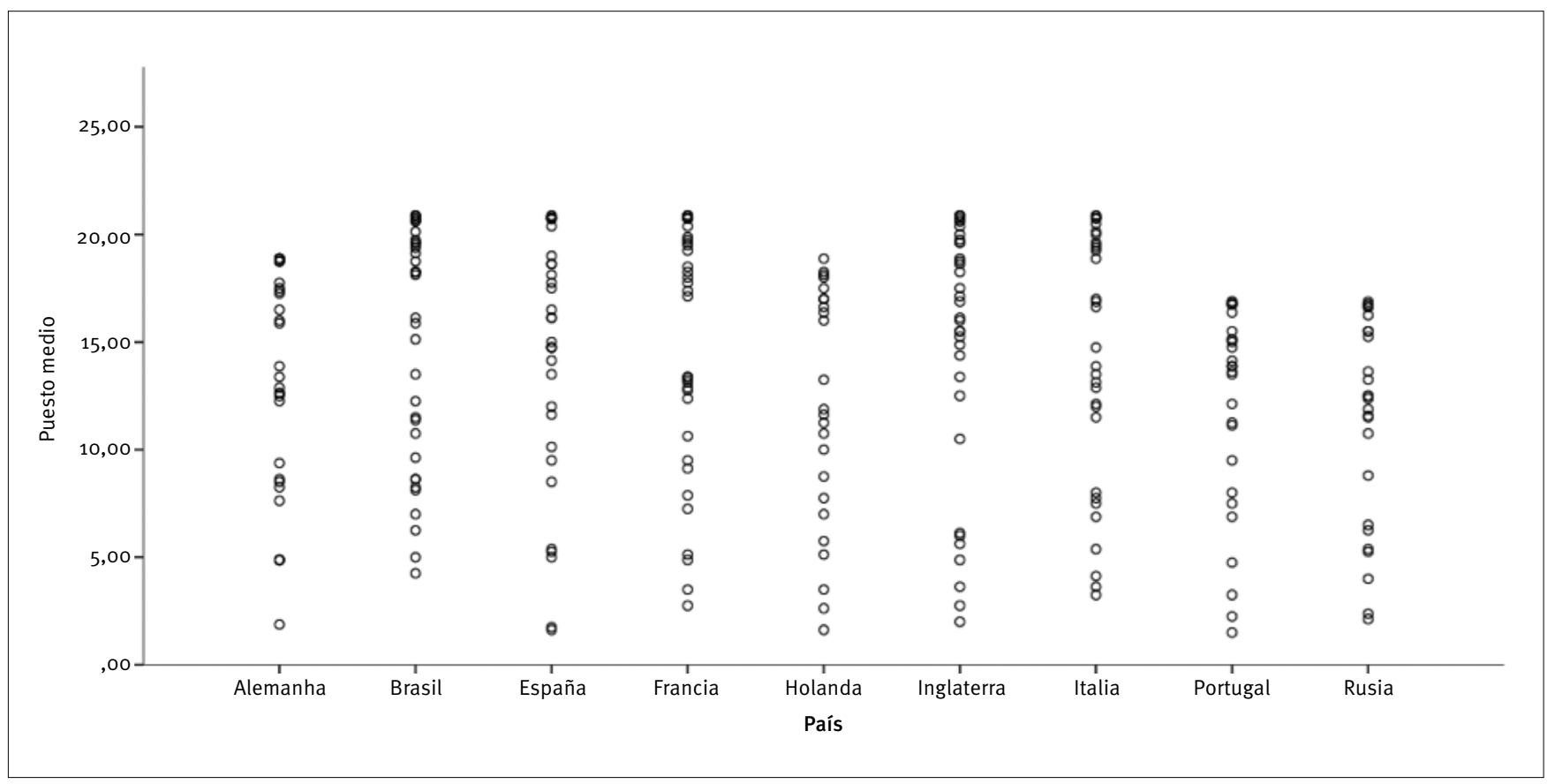

Aunque a través del Gráfico 3 no se pueda inferir sobre el balance competitivo de las ligas, la observación de los puestos medios de los clubes proporciona consideraciones sobre el comportamiento de sus equipos. Se aprecia que en el Campeonato Brasileiro los puntos son más cercanos, lo que demuestra la gran oscilación en la clasificación final del torneo. En la Liga BBVA española se evidencia la presencia de dos clubes mucho más fuertes que los demás, así como otros tres equipos que frecuentemente disputaron plaza a la UEFA Champions League. En la Premier League inglesa y en la Série A italiana se nota claramente dos grupos: siete clubes pelean por el título o la clasificación a la UCL y todos los demás participantes sin mayores objetivos en el campeonato. Sin embargo, en el torneo inglés se demuestra todavía más perceptible. En la Bundesliga alemana se evidencia la presencia de un equipo con desempeño muy superior a los demás, así como otros dos equipos destacados. En Francia, Holanda, Portugal y Rusia los grupos dominantes no han sido tan destacados como en las demás ligas europeas. No obstante, la presencia de clubes con puesto medio cercano al valor 1 , demuestra presencia de dominio deportivo durante el período evaluado.

De esa manera, en la Tabla 7 se compara el puesto medio (PM) de los equipos que han logrado algún título (T) durante las ocho temporadas analizadas. 
Tabla 7. Número de títulos y Puesto Medio entre 20062007 y 2013-2014

\begin{tabular}{l|l|c|c}
\hline & Club & Títulos & Puesto medio \\
\hline Alemania & Bayern M. & 4 & 1,88 \\
\hline & Borussia D. & 2 & 4,88 \\
\hline & Stuttgart & 1 & 7,63 \\
\hline \multirow{2}{*}{ Brasil } & Wolfsburg & 1 & 8,5 \\
\hline \multirow{5}{*}{} & São Paulo & 3 & 4,25 \\
\hline & Cruzeiro & 1 & 6,25 \\
\hline & Flamengo & 1 & 8,13 \\
\hline & Fluminense & 2 & 8,63 \\
\hline & Corinthians & 1 & 9,63 \\
\hline
\end{tabular}

España

\begin{tabular}{l|l|l|l}
\hline & Barcelona & 4 & 1,63 \\
\hline & R.Madrid & 3 & 1,75 \\
\hline & Atlético & 1 & 5,00 \\
\hline
\end{tabular}

\begin{tabular}{l|l|c|c}
\hline \multicolumn{5}{|l|}{ Francia } & Lyon & 2 & 2,75 \\
\hline & Marseille & 1 & 3,5 \\
\hline & Lille & 1 & 4,88 \\
\hline & Bordeaux & 1 & 5,13 \\
\hline & PSG & 2 & 7,25 \\
\hline & Montpellier & 1 & 13,38 \\
\hline
\end{tabular}

Holanda

\begin{tabular}{l|l|c|c}
\hline & Ajax & 4 & 1,63 \\
\hline & PSV & 2 & 2,63 \\
\hline & Twente & 1 & 3,5 \\
\hline & AZ & 1 & 5,75 \\
\hline
\end{tabular}

Inglaterra

\begin{tabular}{l|l|l|c}
\hline & Man.Utd & 5 & 2 \\
\hline & Chelsea & 1 & 2,75 \\
\hline & Man.City & 2 & 5,63 \\
\hline
\end{tabular}

Italia

\begin{tabular}{l|l|l|l}
\hline & Inter & 4 & 3,25 \\
\hline & Milan & 1 & 3,63 \\
\hline & Juventus & 3 & 5,38 \\
\hline
\end{tabular}

Rusia

\begin{tabular}{l|l|l|l}
\hline & CSKA & 3 & 2,13 \\
\hline & Zenit & 3 & 2,38 \\
\hline & Rubin & 2 & 5,25 \\
\hline
\end{tabular}

La Tabla 7 evidencia el dominio de algunos clubes en sus ligas nacionales. El hecho de que un club haya presentado puesto medio cercano a 102 , reconoce que el mismo, aunque no haya sido campeón una temporada, siempre estuvo cercano a los primeros puestos. Por lo tanto, en países como Alemania, España, Holanda, Inglaterra, Portugal y Rusia se notan clubes con desempeño notablemente superior a los demás equipos de su categoría.

Los resultados del presente artículo corroboran los hallazgos de Levy (2011), que a través del HHI ha comparado el Campeonato Brasileiro con nueve ligas europeas y evidenció mejor balance competitivo en el torneo sudamericano durante las temporadas 1997-1998 y 2010-2011. Además, los valores de APD de la liga brasileña confirman los resultados de Drummond et al. (2010) sobre el buen equilibrio en el Campeonato Brasileiro. Al considerar las grandes ligas europeas, los resultados presentados también ratifican los estudios de Goossens (2006), Groot (2008), Flores, Forrest, y Tena (2010), Pawlowski et al. (2010), Criado, García, Pedroche y Romance (2013) y Montes, Sala-Garrido, y Usai (2014) que habían evidenciado disminución en la competitividad en las más recientes temporadas. No obstante, con respeto a la investigación de Criado et al. (2013) se comenta una diferencia observada. En ese estudio los autores, a través de una nueva metodología empleada - complex networks - han destacado un aumento en el equilibrio competitivo en la Bundesliga alemana de la temporada 2011-2012 a la 2012-2013. Sin embargo, la presente investigación ha demostrado el resultado inverso, pues se notó gran desequilibrio en dicha temporada, una vez que el club campeón ha logrado el título con 25 puntos más que el segundo clasificado. La divergencia de resultados entre las investigaciones puede ser consecuencia de la manera cómo se ha medido el desempeño, pues en Criado et al. (2013) los autores han trabajado con los puestos y en el presente artículo se han usado los puntos totales al final de cada competición.

\section{Confrontación con modelos tradicionales}

Para comprobar la validez del modelo desarrollado en la presente investigación, se ha comparado a otros dos métodos tradicionales de análisis del balance competitivo (HICB y C4ICB), empleándolos en el Campeonato Brasileiro. A continuación, se presentan los resultados en la Tabla 8 .

Se subraya que mientras en el Accumulated Points Difference se examina la competitividad basada en el máximo desequilibrio posible, los otros dos métodos (HICB y $\mathrm{C}_{4}$ ICB) analizan el balance competitivo en función del máximo equilibrio. Sin embargo, se interpretan los resultados de los tres modelos de una manera similar, pues todos ponen de manifiesto si el torneo ha aumentado o disminuido la competitividad de la liga. 
Tabla 8. Herfindahl Index of Competitive Balance, $C_{4}$ Index of Competitive Balance y Accumulated Points Difference en el Campeonato Brasileiro entre las temporadas 2006 y 2013

\begin{tabular}{c|c|c|c}
\hline Temporada & HICB & C4ICB & APD \\
\hline 2006 & 105,21 & 133,27 & 43,86 \\
\hline 2007 & 104,79 & 124,29 & 52,63 \\
\hline 2008 & 104,39 & 133,62 & 35,09 \\
\hline 2009 & 103,23 & 124,76 & 31,58 \\
\hline 2010 & 105,34 & 132,97 & 37,72 \\
\hline 2011 & 104,11 & 127,54 & 35,09 \\
\hline 2012 & 105,94 & 138,16 & 41,23 \\
\hline 2013 & 105,07 & 129,88 & 49,12 \\
\hline
\end{tabular}

Como ha sido investigado un único torneo, se esperaba similitud en los resultados de los tres métodos. No obstante, algunas diferencias han sido encontradas. Considerando el índice $C_{4} I C B$, la temporada 2007 fue la más equilibrada entre las ocho investigadas. Sin embargo, considerando el HICB la temporada ha tenido equilibrio intermedio y según el APD ese año presentó el peor equilibrio. Se supone que el Accumulated Points Difference refleja mejor la realidad de ese torneo, pues se observa que en la temporada 2007 el equipo campeón (São Paulo) estuvo durante 27 jornadas como líder del torneo hasta lograr el título de la competición. Este es el récord de tiempo en el primer puesto entre los años investigados. Además, el club ha sido el campeón con mayor antelación ( $34^{\underline{a}}$ jornada) desde que se instituyó el modelo Round-Robin duplo en el Campeonato Brasileiro en la temporada 2003 (en 2013 el Cruzeiro también conquistó la Liga Brasileña en la jornada 34). Conjuntamente, la diferencia entre el campeón y el sub-campeón fue de 15 puntos al final de la competición. Además, el equipo con peor desempeño histórico del Campeonato Brasileiro en Round-Robin estuvo en el año de 2007 con solo 17 puntos conquistados en todo el torneo. Por lo tanto, se presume que la temporada 2007 fue, como indica el APD, la de peor balance competitivo durante el período investigado.

Resultado parecido se observó en la temporada 2013. Como se ha comentado previamente, el club campeón también conquistó el título en la $34^{\underline{a}}$ jornada y el equipo con segundo peor desempeño durante los años de 2003-2013 ha disputado ese torneo que conquistó 20 puntos en 38 partidos. Los índices HICB y C4ICB han señalado esa temporada como moderadamente equilibrada durante el período investigado, no obstante el resultado del APD parece más razonable.
Al considerar los valores del Accumulated Points Difference, la temporada con mejor balance competitivo fue la de 2009 - lo mismo se observó a través del Herfindahl Index of Competitive Balance. Ese hallazgo corrobora con Ribeiro y Urrutia (2012), que, basados en las temporadas 2005 y 2006 de la liga brasileña, modelaron el calendario de la liga en 2009 , hecho que resultó, según los autores, en la edición más equilibrada del Campeonato Brasileiro, una vez que en la última jornada habían cuatro clubes con posibilidad de lograr el título.

\section{Efecto en la asistencia y otros factores que influyen en ella}

Los resultados presentados anteriormente ponen de manifiesto que el Campeonato Brasileiro es el torneo más equilibrado entre todos los investigados en este estudio. Sin embargo, se observa que la asistencia media del torneo brasileño en la temporada 2013 ha sido de 14.951 personas, mientras las dos ligas más desequilibradas (Série $A$ italiana y Bundesliga alemana) han presentado números superiores, con 23.365 y 43.173 personas en promedio por partido, respectivamente (Pluri, 2014). Sin embargo, aunque Rottenberg (1956) haya remarcado la necesidad de la incertidumbre del resultado para el mantenimiento del interés por parte de los aficionados, también existen otros factores determinantes de la asistencia en los partidos de fútbol profesional.

Koenigstorfer, Groeppel-Klein, y Kunkel (2010) confirmaron el balance competitivo percibido como el principal determinante para el interés de los aficionados en Alemania e Inglaterra. No obstante, del mismo modo destacaron que el ambiente del estadio y el éxito internacional de los clubes también influyen en el interés de los aficionados. Otros factores también afectan positivamente en la satisfacción del aficionado y en la demanda de entradas, como la imagen del club (Beccarini \& Ferrand, 2006), el efecto novedad de los nuevos estadios (Coates \& Humphreys, 2005), la presencia de jugadores estrellas (DeSchriver, 2007), las estrategias de ventas de entradas (Howard \& Crompton, 2004), el desempeño deportivo (Theodorakis, Alexandris, Tsigilis, \& Karvounis, 2013) y la búsqueda por el título (Pawlowski \& Nalbantis, 2015).

Por lo tanto, se aprecia que el balance competitivo representa un gran poder de atracción de los aficionados. No obstante, la suma de los diversos factores previamente enumerados es la que contribuye a atraer un mayor número de personas a los estadios de fútbol. De esa manera, en el caso concreto del Campeonato Brasileiro, su mayor competitividad representa una ventaja para el torneo. Sin embargo, será necesario desarrollar el mercado, con el mantenimiento de los 
mejores jugadores en sus clubes, perfeccionar el sistema de venta de entradas y servicios complementarios, así como reforzar la imagen de los equipos pues estos factores son determinantes para aumentar la asistencia a los partidos.

\section{Limitaciones y futuras investigaciones}

De la misma manera que los otros métodos de análisis del balance competitivo, el indicador propuesto en este estudio presenta limitaciones. La principal restricción del modelo es que ha sido desarrollado específicamente para la comparación de ligas de fútbol en formato de doble Round-Robin. Esto implica que para poder ser empleado en otros campeonatos o deportes será necesario realizar ajustes para adaptarlos a cada realidad.

Aquí se ha comparado el Accumulated Points Difference con otras dos fórmulas tradicionales de análisis del balance competitivo: Herfindahl Index of Competitive Balance (HICB) y $\mathrm{C}_{4}$ Index of Competitive Balance $\left(\mathrm{C}_{4} \mathrm{ICB}\right)$. Se entiende que se ha alcanzado el objetivo propuesto pues se ha confirmado la validez del modelo desarrollado. Sin embargo, se estimula la comparación del APD con los demás métodos existentes de análisis de la competitividad confrontándolos en distintas ligas, pues solo se lo ha probado en el Campeonato Brasileiro.

El análisis de la dinámica del balance competitivo se presenta como un importante objeto de estudio para nuevas investigaciones. Como destacaron Fort y Maxcy (2003), es necesario el desarrollo de estudios que analicen la relación del balance competitivo con las políticas adoptadas por cada liga de fútbol y la percepción de los aficionados sobre todo ese contexto. De esa manera, comprender los factores que determinan el aumento o la disminución de la competitividad de las ligas de fútbol se presenta como fundamental para el control deportivo en cada país. Así, se podrán generar alternativas para el mantenimiento o, incluso, aumento del interés por parte de aficionados y los demás stakeholders de esa industria.

Son notorios los problemas que afectan al fútbol brasileño. Los clubes pasan por problemas financieros y de gestión, pero también se observan discusiones respeto del mercado interno. Existe debate sobre el formato competitivo del Campeonato Brasileiro, el mantenimiento de los Campeonatos Estaduais, la creación de la Primeira Liga, así como políticas deportivas sobre Financial Fair Play, la financiación de las deudas y el reparto de ingresos televisivos. Como se destaca en la literatura, todos estos elementos están condicionados por el balance competitivo. Por lo tanto, se espera que la presente investigación estimule nuevos estudios en el escenario deportivo brasileño, analizando los efectos que cada uno de estos factores podrán generar al fútbol en Brasil.

\section{CONCLUSIONES}

Al final de la presente investigación, se concluye que el modelo planteado permite comparar el balance competitivo de torneos de fútbol de formato Round-Robin con distinto número participantes. Se ha evidenciado el buen equilibrio competitivo en el Campeonato Brasileiro en paralelo a la Liga BBVA española, Erevidive holandesa, Premier League inglesa, Serie A italiana y Primeira Liga portuguesa. Sin embargo, no se ha observado diferencias estadísticamente significativas entre los campeonatos europeos, presentando así niveles similares de equilibrio.

Se destaca que el estudio no ha tenido por objetivo evaluar y comparar la calidad de los torneos, de los clubes y tampoco de los jugadores, sino el balance competitivo existente en las ligas investigadas. Se observa en la literatura una gran cantidad de modelos de análisis de la competitividad y en este artículo se ha presentado uno más, pero considerando el desequilibrio máximo de cada liga para poder compararlas, aunque tengan distinto número de participantes. De esta manera, se espera que la presente investigación contribuya al desarrollo teórico y metodológico del tema. El uso de la fórmula elaborada permite analizar la competitividad en una perspectiva distinta a la de los modelos anteriormente desarrollados y posibilita nuevas consideraciones sobre el equilibrio de las ligas de fútbol.

\section{NOTA DE LOS AUTORES}

Este estudio comprende los resultados de la investigación desarrollada en el International Laboratory of Intangible-driven Economy (ID Lab) en la National Research University Higher School of Economics' Basic Research Program.

Los autores agradecen a los editores y revisores anónimos por sus comentarios en el proceso de revisión.

\section{REFERENCIAS}

Barajas, A. (2010). Competitive balance and concentration ratios in economic terms in Liga BBVA. Working Paper SSRN. Recuperado de http://ssrn.com/abstract $=2283411$. 
Beccarini, C., \& Ferrand, A. (2006). Factors affecting soccer clubs season tickets holder's satisfaction: The influence of club image and fan's motives. European Sport Management Quarterly, 6(1), 1-22. doi:10.1080/16184740600799154

Booth, R. (2004). The economics of achieving competitive balance in the Australian Football League, 1897-2004. Economic Society of Australia, 23(4), 325-344. doi:10.1111/j.1759-3441.2004.tboo986.x

Budzinski, 0. (2012). The institutional framework for doing sports business: Principles of EU competition policy in sports markets. International Journal of Sport Management and Marketing, 11(1-2), 44-72. doi:10.1504/ijsmm.2012.045485

Buraimo, B., \& Simmons, R. (2008). Do sports fans really value uncertainty of outcome: Evidence from the English Premier League. International Journal of Sports Finance, 3, 146-155.

Cairns, J. P., Jennett, N., \& Sloane, P. (1986). The economics of professional teams sports: A survey of theory and evidence. Journal of Economic Studies, 13(1), 3-80. doi:10.1108/eboo2618

Coates, D., \& Humphreys, B. R. (2005). Novelty effect of new facilities on attendance at professional sporting events. Contemporary Economic Policy, 23(3), 436-455. doi:10.1093/cep/byio33

Criado, R., García, E., Pedroche, F., \& Romance, M. (2013). A new method for comparing rankings through complex networks: Model and analysis of competitiveness of major European soccer leagues. CHAOS, 23(043114), 1-10. doi:10.1063/1.4826446

Curran, J., Jennings, I., \& Sedgwick, J. (2009). 'Competitive balance' in the top level of English football, 1948-2009: An absent principle and a forgotten ideal. The International Journal of the History of Sport, 26(11), 1735-1747. doi:10.1080/09523360903133087

Czarnitzki, D., \& Stadmann, G. (2002). Uncertainty of outcome versus reputation: Empirical evidence for the First German Football Division. Empirical Economics, 27(1), 101-112. doi:10.1007/s181-002-8361-7

DeSchriver, T. D. (2007). Economics and finance much adieu about Freddy: Freddy Adu and attendance in Major League Soccer. Journal of Sport Management, 21(3), 438-451.

Dobson, S., \& Goddard, J. (2011). The economics of football. Cambridge: Cambridge University Press.

Drummond, L., Araújo, A. F., Jr., \& Shikida, C. D. (2010). Campeonato Brasileiro de Futebol e balanço competitivo: Uma análise do período 1971-2009. Revista Brasileira de Futebol, 3(2), 73-87.

Eckard, E. W. (1998). The NCAA cartel and competitive balance in college football. Review of Industrial Organization, 13(3), 347-369.

El-Hodiri, M., \& Quirk, J. (1971). An economic model of a professional sports league. Journal of Political Economy, 79(6), 1302-1319.

Evans, R. (2014). A review of measures of competitive balance in the 'analysis of competitive balance' literature [Research Paper Series, Vol. 7, ‥ ${ }^{2}$ ]. University of London, Birkbeck Sport Business Centre, London.

Flores, R., Forrest, D., \& Tena, J. D. (2010). Impact on competitive balance from allowing foreign players in a sports leagues: Evidence from European soccer. Kylos, 63(4), 546-557. doi:10.1111/j.1467$6435.2010 .00487 . x$

Forrest, D., \& Simons, R. (2002). Outcome uncertainty and attendance demand in sport: The case of English soccer. The Statistician, 51(2), 229-241. doi:10.1111/1467-9884-00314

Fort, R. (2007). Comments on 'Measuring Parity'. Journal of Sports Economics, 8(6), 642-651. doi:10.1177/1527002506298122
Fort, R., \& Quirk, J. (1995). Cross-subsidizations, incentives, outcome in professional teams sports leagues. Journal of Economic Literature, 33(3), 1265-1299.

Fort, R., \& Maxcy, J. (2003). Competitive balance in sports leagues: An introduction. Journal of Sports Economics, 4(2), 154-160. doi:10.1177/1527002503004002005

Franck, E. (2014). Financial fair play in European football clubs: What is it all about? International Journal of Sports Finance, 9(3), 193-217.

Goossens, D. R., \& Spieksma, F. C. R. (2012). Soccer schedules in Europe: An overview. Journal of Scheduling, 15(5), 641-651. doi:10.1007/ s10951-011-0238-9

Goossens, K. (2006). Competitive balance in European football: Comparison by adapting measures: National measure of Seasonal Imbalance and Top 3. Rivista di Diritto ed Economia Dello Sport, 2(2), 77-122.

Groot, L. F. M. (2008). Economics, uncertainty and European football: Treds in competitive balance. Cheltenham: Edward Elgar.

Haan, M., Koning, R. H., \& Witteloostuijn, A. (2007). Competitive balance in national European soccer competitions. In J. Albert, \& R. H. Koning (Eds.). Statistical thinking in sports (Chap. 4, pp. 63-76). London: Taylor and Francis.

Hausman, J., \& Leonard, G. (1997). Superstars in the National Basketball Association: Economic value and policy. Journal of Labor Economics, 15(4), 586-624. doi:10.1086/209839

Howard, D., \& Crompton, J. (2004). Tactics used by sports organizations in the United States to increase ticket sales. Managing Leisure, 9(2), 87-95. doi:10.1080/13606710410001709617

Humphreys, B. (2002). Alternative measures of competitive balance in sports leagues. Journal of Sports Economics, 3(2), 133-148. doi:10.1177/152700250200300203

Janssens, P., \& Késenne, S. (1987). Belgium football attendances. Tijdschrift voor Economie en Management, 32(3), 305-315.

Késenne, S. (2000). Revenue sharing and competitive balance in professional team sports. Journal of Sports Economics, 1(1), 56-65. doi:10.1177/152700250000100105

Koenigstorfer,J.,Groeppel-Klein,A., \& Kunkel,T. (2010).Theattractiveness of national and international football leagues: Perspectives of fans of "star clubs" and "underdogs". European Sport Management Quarterly, 10(2), 127-163. doi:10.1080/16184740903563406

Koning, R. H. (2000). Balance in competition in Dutch soccer. The Statistician, 49(3), 419-431.

Levin, R., Mitchell, G., Volcker, P., \& Will, G. (2000). The report of the independent members of the Comissioner's Blue Ribbon Panel on baseball economics. Recuperado de http://www.mlb.com/mlb/ downloads/blue_ribbon.pdf.

Levy, P. (2011). Sports administration: An examination of the competitive balance concept through European and Brazilian domestic soccer leagues comparison. Trabajo Fin de Máster, Escola de Administração de Empresas de São Paulo, Fundação Getulio Vargas, Brasil.

Michie, J., \& Oughton, C. (2004). Competitive balance in football: Trends and effects [Research Paper 2014, № 2]. University of London, Football Governance Research Center, London.

Montes, F., Sala-Garrido, R., \& Usai, A. (2014). The lack of balance in the Spanish first division football league. European Sport Management Quartely, 14(3), 282-298. doi:10.1080/16184742.2014.898678 
Müller, C., Lammert, J., \& Hovemann, G. (2012). The financial fair play regulations of UEFA: An adequate concept to ensure the long-term viability and sustainability of European club football? International Journal of Sport Finance, 7, 117-140.

Neale, W. (1964). The peculiar economics of professional sports. Quarterly Journal of Economics, 78(1), 1-14.

Noll, R. (1988). Professional basketball [Working Paper № 144]. Stanford University, Studies in Industrial Economics, Stanford.

Owen, P. D. (2010). Limitations of the relative standard deviation of win percentages for measuring competitive balance in sports leagues. Economics Letters, 109(1), 38-41. doi:10.1016/j.econlet.2010.07.012

Owen, P. D., Ryan, M., \& Weatherspoon, C. R. (2007). Measuring competitive balance in professional team sports using the HerfindahlHirschman index. Review of Industrial Organization, 31(4), 289-302.

Pawlowski, T., \& Anders, C. (2012). Stadium attendance in German professional football: The (un)importance of uncertainty of outcome reconsidered. Applied Economics Letters, 19(16), 1553-1556. doi:10.1 080/13504851.2011.639725

Pawlowski, T., Breuer, C., \& Hovemann, A. (2010). Top club's performance and the competitive situation in European domestic football competitions. Journal of Sports Economics, 11(2), 186-202. doi:10.1177/1527002510363100

Pawlowski, T., \& Nalbantis, G. (2015). Competition format, championship uncertainty and stadium attendance in European football: A small league perspective. Applied Economics, 47(38), 4128-4139. doi:10.1 080/00036846.2015.1023949

Peel, D. A., \& Thomas, D. A. (1988). Outcome uncertainty and the demand for football: An analysis of match attendance in the English football league. Scottish Journal of Political Economy, 35(3), 242-249. doi:10.1111/j.1467-9485.1988.tbo1049.x

Peel, D. A., \& Thomas, D. A. (1992). The demand for football: Some evidence on outcome uncertainty. Empirical Economics, 17(2), 323331. doi:10.1007/bfo1206291

Peeters, T., \& Szymanski, S. (2014). Financial fair play in European football. Economic Policy, 29(78), 343-390.

Pluri. (2014). $3^{0}$ ranking mundial de público nos estádios 2013/14. São Paulo: Fernando Ferreira. Recuperado de http://www.pluriconsultoria.com.br/uploads/relatorios/PLURI\%20especial\%20-\%20 ranking $\% 2$ mundial\%20de $\% 20$ publico $\% 2$ onos\%2oestadios $\% 20$ 2014\%20-\%20campeonatos.pdf.
Preuss, H., Haugen, K., \& Schubert, M. (2014). UEFA financial fair play: The curse of regulation. European Journal of Sport Studies, 2(1), 33-51. doi:10.12863/ejssax2x1-2014x1

Quirk, J., \& Fort, R. D. (1991). Pay dirty: The business of professional teams sports. Princeton, New Jersey: Princeton University Press.

Ribeiro, C. C., \& Urrutia, S. (2012). Scheduling the Brazilian soccer tournament: Solution approach and practice. Interfaces, 42(3), 260272. doi:10.1287/inte.1110.0566

Rodríguez, P. (2012). La economía del deporte. Estudios de Economía Aplicada, 30(2), 1-32.

Rottenberg, S. (1956). The baseball player's labour market. Journal of Political Economy, 64(3), 242-258.

Scully, G. W. (1989). The business of major league baseball. Chicago, IL: University of Chicago Press.

Szymanski, S. (2003). The economic design of sporting contests. Journal of Economic Literature, 41(4), 1137-1187. doi:10.1257/002205103771800004

Szymanski, S. (2014). Fair is foul: A critical analysis of UEFA financial fair play. International Journal of Sports Finance, 9(3), 218-229.

Szymanski, S., \& Kuypers, T. (1999). Winners and losers: The business strategy of football. London: Viking.

Theil, H. (1967). Economics and information theory. Chicago: McGrawHill.

Theodorakis, N. D., Alexandris, K., Tsigilis, N., \& Karvounis, S. (2013). Predicting spectators' behavioural intentions in professional football: The role of satisfaction and service quality. Sport Management Review, 16(1), 85-96. doi:10.1016/j.smr.2012.05.004

Trandel, G. A., \& Maxcy, J. G. (2011). Adjusting winning percentage standard deviations and a measure of competitive balance for home advantage. Journal of Quantitative Analysis in Sports, 7(1), 1-17. doi:10.2202/1559-0410.1297

Vrooman, J. (1995). A general theory of professional sports leagues. Southern Economic Journal, 61(4), 971-990. doi:10.2307/1060735

Vrooman, J. (1996). The baseball players labour market reconsidered. Southern Economic Journal, 63(2), 339-360. doi:10.2307/1061172

Zimbalist, A. (2002). Competitive balance in sports leagues: An introduction. Journal of Sports Economics, 3(2), 111-121. doi:10.1177/152700250200300201 